\title{
Construção e validação de material educativo sobre tratamento farmacológico da Leucemia Mieloide Crônica
}

\author{
Vanessa Casagrande \\ Doutoranda em Pesquisa e Desenvolvimento (Biotecnologia Médica) (UNESP) \\ Mestre em Ensino em Saúde (FAMEMA) \\ $\triangle$ vancasagrande@yahoo.com \\ Maria Angélica Spadella \\ Doutora em Biologia Celular e Estrutural (UNICAMP) \\ Docente do Programa de Pós-graduação em Saúde e Envelhecimento \\ Disciplina de Embriologia Humana (FAMEMA) \\ $\triangle$ maspadella@gmail.com \\ Eduardo Federighi Baisi Chagas \\ Programa de Pós-Graduação em Interações Estruturais e Funcionais em Reabilitação (UNIMAR) \\ Docente do Programa de Pós-Graduação (FAMEMA) \\ 凶efbchagas@gmail.com \\ Osni Lázaro Pinheiro \\ Doutor em Ginecologia e Obstetrícia (UNESP) \\ Docente do Programa de Pós-graduação Ensino em Saúde e da Disciplina de Farmacologia (FAMEMA) \\ 凶osnilp@terra.com.br
}

Recebido em 28 de outubro de 2020

Aceito em 16 de novembro de 2021

\section{Resumo:}

Elaborar um material educativo com enfoque no tratamento da Leucemia Mieloide Crônica. Inicialmente verificou-se a adesão ao tratamento medicamentoso dos pacientes do Ambulatório de Onco-hematologia ( $\mathrm{n}=36)$ aplicando a Escala de Morisky. Em seguida foi elaborada uma versão preliminar do material educativo com participação dos pacientes $(n=10)$ e profissionais da área de saúde $(\mathrm{n}=10)$. Os participantes contribuíram na definição dos temas e posteriormente na avaliação do material educativo. Foi observado que a adesão dos pacientes ao tratamento merece investimentos e os avaliadores trouxeram importantes contribuições para a construção de um material educativo sucinto, claro e com ilustrações. Os pacientes com Leucemia Mieloide Crônica merecem atenção e o material validado poderá ser utilizado como ferramenta para a realização de ações de "Educação em Saúde", podendo auxiliar na otimização das taxas de adesão ao tratamento farmacológico.

Palavras-chave: Materiais educativos e de divulgação, Assistência farmacêutica, Proteínas tirosina quinases, Leucemia mielogênica crônica BCR-ABL positiva.

\section{Development and validation of pharmacological treatment educational material for Chronic Myeloid Leukemia}

\begin{abstract}
:
To create educational material focusing on the treatment of patients with Chronic Myeloid Leukemia. Patients from the Onco-Hematology outpatient clinic were initially enrolled in the pharmacological treatment of CML using the Morisky Scale $(n=36)$. Next, a first version of the educational material was created with the help of patients $(n=10)$ and health professionals $(n=10)$. They helped define themes
\end{abstract}


and evaluate the educational material. A second version of the material was created and sent to health professionals for a final assessment. The outcome showed that the rate of patient adherence to treatment deserve an increase on investment and, also, the evaluators brought important contribution to the development of an illustrated, succinct and clear educational material. That patients with CML deserve attention and the validated material can be used as a tool in health education practices, promoting improvements in adherence rates to the pharmacological treatment of CML.

Keywords: Educational and promotional materials, Pharmaceutical services, Protein-tyrosine kinases, Leukemia, myelogenous, chronic, BCR-ABL positive.

\title{
Construcción y validación de material educativo sobre tratamiento farmacológico de la Leucemia Mieloide Crónica
}

\begin{abstract}
Resumen:
Desarrollar material educativo con un enfoque en el tratamiento de la leucemia mieloide crónica. Inicialmente, se verificó la adherencia al tratamiento farmacológico de los pacientes del Ambulatorio de Oncohematología $(n=36)$ aplicando la Escala de Morisky. Luego, se elaboró una versión preliminar del material educativo con la participación de pacientes $(n=10)$ y profesionales de la salud $(n=10)$. Los participantes contribuyeron a la definición de temas y posteriormente a la evaluación del material educativo. Se observó que la adherencia de los pacientes al tratamiento merece inversiones y los evaluadores aportaron importantes contribuciones para la construcción de un material educativo sucinto, claro e ilustrado. Los pacientes con Leucemia Mieloide Crónica merecen atención y el material validado puede ser utilizado como una herramienta para realizar acciones de "Educación para la Salud", que pueden ayudar a optimizar las tasas de adherencia al tratamiento farmacológico.

Palabras clave: Materiales Educativos y de Divulgación, Servicios Farmacéuticos, Proteínas Tirosina Quinasas, Leucemia Mielógena Crónica BCR-ABL Positiva.
\end{abstract}

\section{INTRODUÇÃO}

Estratégias educativas com o apoio de folders e cartilhas podem ser utilizadas para a execução de ações em saúde que tenham o propósito de melhorar a compreensão dos pacientes em relação ao seu estado de saúde, assim como esclarecer as particularidades inerentes ao tratamento medicamentoso.

Em estudo realizado com mães de neonatos submetidos à fototerapia foi destacada a importância dos materiais educativos para esclarecer os benefícios da fototerapia no tratamento da icterícia neonatal. As mães relataram que antes da realização das atividades com o apoio do material educativo havia insegurança em relação ao uso do berço com luz ultravioleta, pois as informações recebidas dos profissionais de saúde eram divergentes (IVO et al., 2017).

Materiais educativos também podem ser utilizados em situações de saúde de maior complexidade. Neste sentido foi desenvolvido um trabalho que tinha o propósito de elaborar 
uma cartilha para auxiliar pacientes com insuficiência renal, submetidos à hemodiálise. A validação do material em relação à compreensibilidade da linguagem e ao conteúdo foi realizada pelos próprios pacientes (MEDEIROS et al., 2016).

A participação dos pacientes na construção dos materiais educativos é um recurso bastante utilizado, pois o conhecimento do paciente em relação ao problema de saúde que apresenta favorece a delimitação do conteúdo (SANTIAGO et al., 2020).

A hematologia representa uma das áreas da medicina nas quais as ações em saúde representam aspectos importantes para melhorar a compreensão dos pacientes sobre sua doença e com isso favorecer a adesão ao tratamento.

A Leucemia Mieloide Crônica (LMC) é uma doença hematológica que representa 15\% das leucemias que acometem indivíduos adultos (Network, 2020). O tratamento medicamentoso da LMC aumenta a sobrevida dos pacientes e uma das vantagens é a possibilidade de utilização dos medicamentos por via oral, porém, a adesão à terapia oral tem variado bastante (ROESE; FONTANA; PEREIRA, 2018).

Em estudo realizado com portadores de LMC foi verificado que o imatinibe, um destes fármacos administrados por via oral, não era utilizado adequadamente em decorrência de informações insuficientes para os pacientes, tanto em relação a sua doença, como também ao tratamento (BRECCIA et al., 2015).

Portanto, ainda existe a necessidade de investimentos dos profissionais de saúde para melhorar a adesão dos pacientes a este tratamento.

Os objetivos deste estudo perpassaram pela verificação do grau de adesão dos pacientes portadores de LMC em relação ao tratamento medicamentoso e elaboração de um material educativo para este público alvo.

\section{METODOLOGIA}

O presente estudo foi de natureza observacional, com coleta de dados de maneira prospectiva com utilização de questionários. 
O estudo foi realizado no Ambulatório de Onco-Hematologia do Hemocentro de uma faculdade pública do interior do Estado de São Paulo. A amostra deste estudo foi constituída por pacientes deste ambulatório com diagnóstico de LMC e que estavam em seguimento terapêutico farmacológico por via oral com medicamentos conhecidos como "inibidores da tirosina quinase". Os pacientes participaram na etapa que consistiu da verificação da taxa de adesão ao tratamento $(n=36)$ e uma parte destes na validação do material educativo $(n=10)$. Também participaram da validação do material educativo os profissionais da área da saúde com expertises nas áreas de farmacologia ou onco-hematologia $(n=10)$.

$\mathrm{Na}$ primeira etapa deste estudo foi verificada a adesão dos pacientes com LMC ao tratamento com medicamentos inibidores da enzima tirosina quinase (Imatinibe, Dasatinibe e Nilotinibe). A adesão ao tratamento foi realizada por meio da aplicação da Escala de Adesão Terapêutica de oito itens de Morisky (MMAS-8) adaptada ao contexto da LMC (MORISKY et al., 2008).

Os pacientes com escore 8 (oito) foram considerados altamente aderentes ao tratamento. A adesão foi considerada mediana para os que apresentaram escore entre 7 (sete) e 6 (seis) e baixa adesão para os que apresentaram escore menor que 6 (seis) (MORISKY et al., 2008).

Para definição dos conteúdos do material educativo, foi realizado um levantamento de dados na literatura, para a construção de um questionário contendo assertivas sobre os temas que deveriam ser abordados no material educativo.

Após sua construção, o questionário foi enviado para um grupo de juízes especialistas e também para um grupo de pacientes com o objetivo de validar a proposta inicial dos temas sobre LMC. Cada assertiva foi seguida de uma escala do tipo Likert com quatro pontos: discordo totalmente, discordo, concordo e concordo totalmente (SAKO et al., 2018). Também foi disponibilizado um campo aberto no qual os participantes puderam expressar livremente suas opiniões sobre o tema.

Os itens do questionário foram considerados validados quando a porcentagem de concordância entre os avaliadores atingiu minimamente 80\% (PASQUALI, 1997). O conteúdo dos campos abertos do questionário foi analisado e as sugestões consideradas pertinentes foram incorporadas na versão inicial do material educativo. 
Após a análise dos dados provenientes dos instrumentos preenchidos pelos pacientes e pelos profissionais de saúde foi elaborada uma primeira versão do material educativo sobre o tratamento farmacológico da LMC.

Esta versão foi entregue impressa ou enviada por email para os mesmos profissionais de saúde e pacientes que participaram na etapa anterior.

Além do material educativo, os participantes receberam também um questionário de avaliação contendo assertivas relacionadas ao conteúdo, linguagem, ilustrações, layout, motivação e cultura, segundo adaptação do modelo proposto por Sousa (SOUSA; TURRINI, 2012). Cada assertiva foi seguida de uma escala do tipo Likert com quatro pontos e também um campo aberto.

A validação do material educativo também foi realizada de acordo com o percentual de concordância e os itens foram validados quando a porcentagem de concordância atingiu minimamente 80\% (PASQUALI, 1997).

Os conteúdos presentes no campo "comentários adicionais" foram analisados e as sugestões consideradas pertinentes foram incorporadas ao material educativo.

Em seguida houve a confecção da segunda versão do material educativo, o qual foi submetido aos especialistas, que validaram novamente o conteúdo, linguagem, ilustrações, layout, motivação e cultura.

A análise estatística da distribuição de frequência das variáveis qualitativas relacionadas ao questionário de Morisky foi realizada utilizando o teste Exato de Fisher. Todas as análises foram desenvolvidas com o software SPSS versão 19.0 for Windows, sendo adotado nível de significância de $5 \%$.

O presente estudo foi aprovado pelo Comitê de Ética em Pesquisa Envolvendo Seres Humanos da Faculdade de Medicina de Marília (Parecer nº 1.850.090 e CAAE nº 62006616.0.0000.5413).

\section{RESULTADOS E DISCUSSÃO}

A adesão foi verificada em 36 pacientes atendidos no período de maio a novembro de 2017. 0 tempo de tratamento para a maioria dos pacientes no momento de aplicação do questionário variou de 6 a 10 anos (57\%). 
De uma maneira geral, os pacientes eram brancos $(69,4 \%)$ do sexo feminino $(61,1 \%)$, com idade inferior a 60 anos $(55,6 \%)$, casados $(55,6 \%)$ e com no máximo dois filhos $(63,9 \%)$. Os pacientes mencionaram que possuíam pouca ou nenhuma escolaridade $(52,8 \%)$, eram de religião evangélica (52,8\%) e apresentavam renda familiar máxima de R\$2.000,00 (66,7\%).

Embora a maioria dos pacientes deste estudo tenha se mostrado altamente aderente ao tratamento prescrito $(55,6 \%)$, cabe destacar que houve irregularidade em relação à completa adesão ao tratamento em aproximadamente metade $(44,4 \%)$ da populaçao deste estudo. (Tabela 1).

Tabela 1 - Adesão dos pacientes em seguimento terapêutico via oral com inibidores da tirosina quinase $(n=36)$

\begin{tabular}{lccc}
\hline Nível de adesão & $\mathbf{n}$ & $\mathbf{\%}$ & p-valor \\
\hline Alta adesão & 20 & 55,6 & \multirow{2}{*}{0,505} \\
Falha na adesão* & 16 & 44,4 &
\end{tabular}

Legenda: *Falha na adesão = Média + baixa adesão.

Fonte: Própria.

Considerando as características da doença dos pacientes deste estudo, onde a ausência do medicamento pode causar piora do quadro clínico, é recomendado que o tratamento atinja níveis de excelência em relação a adesão, o que não ocorreu neste estudo. Portanto, os resultados mostram que existe a necessidade de implementação de estratégias educativas para reverter estes resultados.

Pelo fato de a LMC representar uma doença que se manifesta com poucos sintomas, o paciente pode apresentar dificuldade de permanecer motivado para continuar o tratamento, causando irregularidades na adesão (SIMONEAU, 2013).

A falta de informação sobre a doença é outro fator que pode interferir na adesão do paciente ao tratamento. Dessa forma, é essencial propiciar estratégias para assegurar a continuidade da terapia farmacológica e obter todos os benefícios da mesma (HAMERSCHLAK et al., 2015).

Um estudo realizado com pacientes em fase crônica da LMC mostrou que aqueles que foram considerados não aderentes, com interrupção do tratamento por mais de uma semana, apresentaram uma diminuição da sobrevida, com comprometimento dos parâmetros 
hematológico, citogenético e molecular, além de progressão da doença para as fases acelerada/crise blástica e morte (GANESAN et al., 2011).

Dentro da amostra de pacientes que participou deste estudo na etapa da verificação da adesão ao tratamento, estão aqueles que atuaram como avaliadores do material educativo. Estes apresentaram idade média de 49,4 \pm 15,3 anos (média \pm desvio padrão), $60 \%$ com ensino médio completo e $40 \%$ destes com formação superior e com tempo de tratamento no momento de aplicação do questionário entre 2 a 10 anos (Quadro 1).

Quadro 1 - Caracterização dos pacientes que participaram da validação do material educativo

\begin{tabular}{|c|c|c|c|c|}
\hline Paciente & Idade & Escolaridade & Profissão & $\begin{array}{c}\text { Tempo em } \\
\text { tratamento }\end{array}$ \\
\hline $\mathbf{1}$ & 59 & E. Médio completo & Serviços gerais & 08 \\
\hline $\mathbf{2}$ & 53 & Superior completo & Professora & 10 \\
\hline $\mathbf{3}$ & 48 & Superior completo & Auxiliar jurídico/vendedora & 02 \\
\hline $\mathbf{4}$ & 75 & Superior completo & Aposentada & 07 \\
\hline $\mathbf{5}$ & 41 & E. Médio completo & Vigilante patrimonial & 02 \\
\hline $\mathbf{6}$ & 46 & E. Médio completo & Motorista & 02 \\
\hline $\mathbf{7}$ & 27 & E. Médio completo & Operadora de caixa & 08 \\
\hline $\mathbf{8}$ & 62 & Superior completo & Comerciante & 08 \\
\hline $\mathbf{9}$ & 26 & E. Médio completo & Pintor & 03 \\
\hline $\mathbf{1 0}$ & 57 & E. Médio completo & Auxiliar de enfermagem & 07 \\
\hline
\end{tabular}

Fonte: Própria.

O grupo de profissionais de saúde que atuou na validação do material educativo foi proveniente de diferentes instituições de ensino superior e serviços especializados na área de onco-hematologia.

Participaram do estudo médicos hematologistas (2), farmacêuticos (7) e enfermeira da área de hematologia e quimioterapia (1). A idade destes profissionais foi de 41,7 $\pm 7,3$ anos (média \pm desvio padrão) com experiência na área de atuação de $15 \pm$ 7,5 anos (média \pm desvio padrão). Em relação a titulação, $40 \%$ possuem mestrado, $40 \%$ doutorado e $20 \%$ são especialistas (Quadro 2). 
Quadro 2 - Caracterização dos profissionais que atuaram como juízes na validação do material educativo

\begin{tabular}{|c|c|c|c|c|}
\hline Juiz & Formação & $\begin{array}{c}\text { Experiência } \\
\text { (anos) }\end{array}$ & Titulação & Área Atuação \\
\hline $\mathbf{1}$ & Farmacêutico & 16 & Doutorado & Farmácia Hospitalar \\
\hline $\mathbf{2}$ & Enfermeira & 10 & Mestrado & Hematologia Quimioterapia \\
\hline $\mathbf{3}$ & Farmacêutico & 15 & Mestrado & $\begin{array}{c}\text { Atenção Farmacêutica e Farmácia } \\
\text { Hospitalar }\end{array}$ \\
\hline $\mathbf{4}$ & Farmacêutico & 17 & Mestrado & Oncologia \\
\hline $\mathbf{5}$ & Médico & 20 & Doutorado & Hematologia \\
\hline $\mathbf{6}$ & Farmacêutico & 33 & Doutorado & $\begin{array}{c}\text { Farmacologia e } \\
\text { Epidemiologia Clínica }\end{array}$ \\
\hline $\mathbf{7}$ & Farmacêutico & 8 & Especialização & Atenção Farmacêutica \\
\hline $\mathbf{8}$ & Farmacêutico & 10 & Mestrado & Oncologia \\
\hline $\mathbf{9}$ & Farmacêutico & 13 & Doutorado & Atenção primária a saúde \\
\hline $\mathbf{1 0}$ & Médico & 8 & Especialização & Hematologia e Hemoterapia \\
\hline
\end{tabular}

$\mathrm{Na}$ etapa que consistiu da definição dos temas para elaboração do material educativo, todos os pacientes que atuaram como avaliadores (100\%) concordaram com os itens que foram propostos para compor o material sobre tratamento de LMC (Tabela 2).

Tabela 2 - Avaliações dos especialistas e profissionais de saúde em relação aos temas que devem ser contemplados no material educativo

\begin{tabular}{lcc}
\hline & \multicolumn{2}{c}{ Concordância (\%) } \\
Pssertivas & Especialistas & Pacientes \\
\hline $\begin{array}{l}\text { 1- A cartilha deve explicar o que é o tratamento } \\
\text { para leucemia }\end{array}$ & 89 & 100 \\
$\begin{array}{l}\text { 2- A cartilha deve informar os efeitos colaterais dos } \\
\text { medicamentos que o paciente toma para leucemia }\end{array}$ & 100 & 100 \\
$\begin{array}{l}\text { 3- A cartilha deve explicar sobre o exame } \\
\text { citogenético de amostras de medula óssea }\end{array}$ & 56 & 100 \\
$\begin{array}{l}\text { 4- A cartilha deve explicar o que o paciente precisa } \\
\text { para retirar seu medicamento na Farmácia do }\end{array}$ & 89 & 100 \\
$\begin{array}{l}\text { Hemocentro } \\
\text { 5- A cartilha deve conter figuras ilustrativas sobre a } \\
\text { leucemia mieloide crônica }\end{array}$ & 89 & 100 \\
$\begin{array}{l}\text { 6- A cartilha deve ter informações sobre as } \\
\text { consequências de não tomar o medicamento para } \\
\text { leucemia }\end{array}$ & 100 & 100 \\
$\begin{array}{l}\text { 7- A cartilha deve explicar como tomar o } \\
\text { medicamento para leucemia }\end{array}$ & 100 & 100 \\
$\begin{array}{l}\text { 8- A cartilha deve explicar sobre as interações do } \\
\text { medicamento para leucemia com os alimentos }\end{array}$ & 100 & 100 \\
\hline $\begin{array}{l}\text { Legenda: (DT) Discordo Totalmente, (D) Discordo,(C) Concordo, (CT) Concordo Totalmente. } \\
\text { Fonte: Própria. }\end{array}$
\end{tabular}


Em relação aos profissionais de saúde, obteve-se 100\% de concordância para as assertivas 2, 6, 7 e 8. Nas assertivas 1, 4 e 5 a concordância foi de 89\%. (Tabela 2).

Entretanto, na avaliação da assertiva 3, que abordava o exame citogenético de células da medula, a concordância para manutenção deste item ocorreu apenas em $56 \%$ das avaliações, não atingindo assim o grau mínimo de concordância adotado por este estudo. Além disso, um dos profissionais de saúde não avaliou esta assertiva (Tabela 2).

A realização de esclarecimentos sobre o diagnóstico da LMC é importante, tendo em vista tratar-se de exames invasivos, como é o caso da coleta de amostras de material proveniente da medula óssea, destinado para exame citogenético (BONAVIGO et al., 2018). Tendo em vista este resultado, optou-se apenas por ilustrar a origem das células sanguíneas na medula óssea a fim de remeter aos motivos pelos quais o exame é colhido no osso esterno ou no quadril (Figura 1).

Figura 1. Ilustração das células sanguíneas na medula óssea

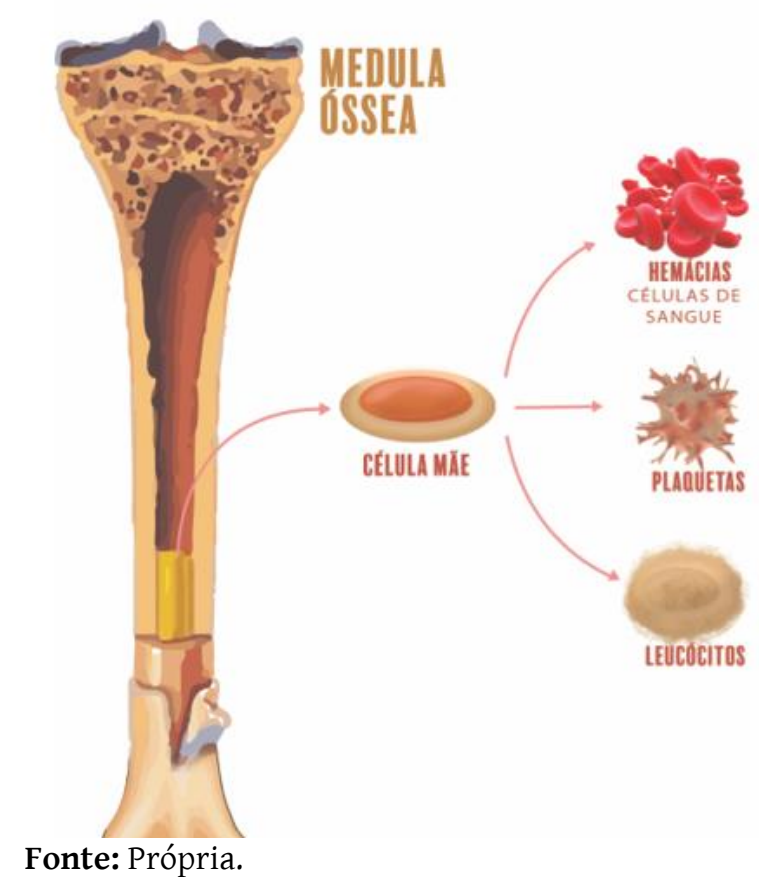

Os campos abertos do questionário de validação dos itens propostos para compor o material educativo foram bastante utilizados pelos juízes. De maneira unânime, os comentários reforçaram a necessidade de esclarecimentos sobre efeitos adversos, consequências da falta de adesão ao tratamento, duração do tratamento, critérios de cura e 
os medicamentos que não devem ser administrados concomitantemente ao tratamento para LMC.

Os medicamentos utilizados para o tratamento da LMC geralmente estão associados a efeitos colaterais. Os mais comuns são fadiga, náusea, diarreia, edema, erupção cutânea, mialgia e artralgia (JABBOUR et al., 2012).

Desta forma, é importante que o material educativo esclareça sobre estes efeitos, enfatizando que os benefícios em relação à remissão da doença são maiores. Esta compreensão poderá favorecer a adesão ao tratamento.

No material educativo os efeitos colaterais mais comuns e as sugestões de medidas não farmacológicas foram abordados para cada medicamento separadamente. Dentre estas medidas foi destacada a importância de reduzir o consumo de sal, realizar exercícios físicos diariamente para diminuir o edema, um pequeno repouso durante o dia para evitar a fadiga e não usar adoçantes para evitar diarreia (SIMONEAU, 2013).

Embora a literatura registre as condutas farmacológicas que devem ser adotadas para minimizar os efeitos colaterais deflagrados pelos medicamentos inibidores da tirosina quinase, foi feita a opção por não abordar estas informações no material educativo, de modo a não estimular a automedicação.

Outra informação incorporada ao material foi a orientação da melhor maneira de se administrar os medicamentos para o tratamento da LMC, em virtude de que alguns agentes farmacológicos para o tratamento da LMC podem ter suas ações modificadas em decorrência de interações com alimentos ingeridos pelo paciente (STEEGMANN et al., 2016). Tendo em vista que os alimentos podem interferir na absorção dos medicamentos, ou desencadear distúrbios gastrointestinais quando ingeridos em jejum, podendo assim interferir na adesão do paciente ao tratamento, optou-se por abordar a melhor forma de ingerir cada medicamento individualmente (SILVA et al., 2017).

No material educativo foi mencionado que o tratamento medicamentoso quando seguido rigorosamente controla os sintomas e impede que a doença evolua e também foram destacados os riscos e contraindicações do transplante de medula óssea (SIMONEAU, 2013; ABBOUR et al., 2012). 
Outro aspecto inicialmente idealizado na proposta do material educativo foi a inclusão de informações sobre a entrega dos medicamentos para os pacientes na farmácia vinculada ao ambulatório de onco-hematologia. Os pacientes relataram que geralmente existem dúvidas a este respeito e os profissionais de saúde destacaram que estas informações melhorariam a adesão ao tratamento. Desta forma foi decidido incorporar na contracapa do material educativo o endereço e horário de funcionamento da farmácia e o horário de entrega dos medicamentos, destacando também os documentos necessários para este procedimento.

Uma vez que a presença de ilustrações em materiais educativos destinados para os usuários do sistema de saúde favorece a compreensão dos conteúdos abordados, pois podem ser utilizados como apoio pedagógico para os profissionais de saúde realizarem suas atividades com os pacientes, deciciu-se recorrer à um ilustrador para criação das imagens e dos personagens e a um diagramador para construir o material em formato de histórias em quadrinhos a fim de facilitar a compreensão dos usuários, e motivar a leitura, visto que o nível de escolaridade pode influenciar na compreensão dos textos (MEDEIROS et al., 2016; IVO et al., 2017).

Neste sentido, a presença de ilustrações associadas a um conteúdo mais objetivo e de fácil assimilação podem ser motivadores para a realização da atividade de educação em saúde (RAMOS; ARAÚJO, 2017).

Com a utilização de todas estas informações foi confeccionada a versão preliminar do material educativo. Este material, nesta versão inicial possuía 14 páginas, com 14 ilustrações.

Após a confecção do material, os profissionais de saúde e pacientes foram convidados para avaliar o produto. O questionário de avaliação da cartilha utilizado foi o mesmo para os profissionais de saúde e a amostra de pacientes, que representam o público alvo deste estudo (Tabela 3). 
Tabela 3 - Avaliação da primeira versão do material didático sobre LMC realizada por pacientes $(n=9)$ e profissionais de saúde $(n=10)$

\begin{tabular}{|c|c|c|}
\hline \multirow{2}{*}{ Itens Avaliados } & \multicolumn{2}{|c|}{ Concordância (\%) } \\
\hline & Pacientes & Especialistas \\
\hline \multicolumn{3}{|l|}{ 1.Conteúdo } \\
\hline $\begin{array}{l}1.10 \text { conteúdo está apropriado ao público alvo (pacientes } \\
\text { com Leucemia Mieloide Crônica) }\end{array}$ & 100 & 90 \\
\hline $\begin{array}{l}1.2 \text { A divisão dos títulos e subtítulos do material são } \\
\text { pertinentes }\end{array}$ & 100 & 90 \\
\hline $\begin{array}{l}1.3 \text { Os trechos chave (trechos em destaque) são pontos } \\
\text { importantes e merecem destaque }\end{array}$ & 100 & 100 \\
\hline $\begin{array}{l}1.4 \text { O conteúdo é suficiente para atender as necessidades } \\
\text { do público alvo (pacientes com Leucemia Mieloide } \\
\text { Crônica) }\end{array}$ & 100 & 100 \\
\hline \multicolumn{3}{|l|}{ 2. Linguagem } \\
\hline $\begin{array}{l}2.10 \text { estilo da redação é compatível com o público alvo } \\
\text { (pacientes com Leucemia Mieloide Crônica) }\end{array}$ & 100 & 80 \\
\hline 2.2 A escrita utilizada é atrativa & 100 & 100 \\
\hline 2.3 A linguagem é clara e objetiva & 100 & 90 \\
\hline \multicolumn{3}{|l|}{ 3. Ilustrações } \\
\hline $\begin{array}{l}3.1 \text { As ilustrações utilizadas são pertinentes com o } \\
\text { conteúdo do material e esclarecem o conteúdo }\end{array}$ & 100 & 90 \\
\hline $\begin{array}{l}\text { 3.2 As ilustrações são claras e transmitem facilidade de } \\
\text { compreensão }\end{array}$ & 100 & 80 \\
\hline $\begin{array}{l}3.3 \text { As legendas das imagens são adequadas e auxiliam o } \\
\text { leitor a compreender a imagem }\end{array}$ & 100 & 90 \\
\hline $\begin{array}{l}3.4 \text { A quantidade de ilustrações está adequada para o } \\
\text { conteúdo do material educativo }\end{array}$ & 100 & 100 \\
\hline \multicolumn{3}{|l|}{ 4. Layout } \\
\hline 4.1 O tipo de letra utilizado facilita a leitura & 100 & 100 \\
\hline $\begin{array}{l}\text { 4.2 As cores aplicadas ao texto são pertinentes e } \\
\text { facilitadoras para a leitura }\end{array}$ & 100 & 100 \\
\hline 4.3 A composição visual está atrativa e bem organizada & 100 & 100 \\
\hline $\begin{array}{l}4.4 \text { O formato (tamanho) do material educativo e o } \\
\text { número de páginas estão adequados }\end{array}$ & 100 & 100 \\
\hline 4.5 A disposição do texto está adequada & 100 & 100 \\
\hline $\begin{array}{l}4.6 \text { O tamanho das letras dos títulos, subtítulos e textos } \\
\text { estão adequados }\end{array}$ & 100 & 100 \\
\hline \multicolumn{3}{|l|}{ 5. Motivação } \\
\hline $\begin{array}{l}5.10 \text { conteúdo está motivador e incentiva a prosseguir } \\
\text { com a leitura }\end{array}$ & 100 & 90 \\
\hline 5.20 conteúdo despertou interesse no leitor & 100 & 100 \\
\hline $\begin{array}{l}5.3 \text { O conteúdo atende as dúvidas, esclarece e educa o } \\
\text { paciente em relação à Leucemia Mieloide Crônica }\end{array}$ & 100 & 80 \\
\hline \multicolumn{3}{|l|}{ 6. Cultura } \\
\hline $\begin{array}{l}6.1 \text { O texto está compatível com o público alvo, (pacientes } \\
\text { com Leucemia Mieloide Crônica) atendendo aos diferentes } \\
\text { níveis de conhecimento }\end{array}$ & 100 & 70 \\
\hline
\end{tabular}

Fonte: Própria. 
As avaliações dos profissionais de saúde apresentaram uma criticidade em relação aos termos técnicos, bem como sugestões de correções ortográficas e de alguns conteúdos e ilustrações. Estas avaliações favoreceram a elaboração de um material educativo de melhor qualidade.

Após as incorporações das sugestões feitas pelos avaliadores na versão preliminar do material educativo, foi concluída a segunda versão deste material, que foi encaminhada para os juízes especialistas, com o objetivo de uma nova apreciação.

O material foi acompanhado de uma carta de considerações para cada juiz. Nesta carta, foram explicados os motivos do não atendimento de algumas sugestões feitas na primeira avaliação do material educativo. Dentre os 10 avaliadores que receberam esta nova versão do material educativo, cinco retornaram suas avaliações, concordando com as adequações realizadas.

Desta forma, foi finalizada a elaboração do material educativo sobre o tratamento medicamentoso da LMC, que em sua versão definitiva possui 14 páginas e 15 ilustrações e foi disponibilizada, com acesso livre, no endereço eletrônico: http://doi.org/10.5281/zenodo.; 3971163 (CASAGRANDE; SPADELLA; PINHEIRO, 2020).

\section{CONCLUSÃO}

O presente estudo reforçou a hipótese inicial da existência de falhas na adesão ao tratamento medicamentoso oral para LMC. Esta falha na adesão subsidia a importância de investimentos em práticas educativas por parte dos profissionais de saúde, para identificar as necessidades dos pacientes com LMC que possam estar associadas com o tratamento medicamentoso. O material validado poderá ser útil como ferramenta para a realização de ações de "Educação em Saúde", que auxiliem na otimização das taxas de adesão ao tratamento farmacológico da LMC.

\section{AGRADECIMENTOS}

O presente trabalho foi realizado com apoio da Coordenação de Aperfeiçoamento de Pessoal de Nível Superior - Brasil (CAPES) - Código de Financiamento 001, a qual agradecemos. 


\section{REFERÊNCIAS}

BONAVIGO, A. G. et al. Comparação entre o diagnóstico citogenético e por biologia molecular das leucemias mieloides crônicas (LMC): uma revisão bibliográfica. RBAC, Rio de Janeiro, v.2, pS47-S50, 2018. Supl. 2.

BRECCIA, M. et al. Adherence and future discontinuation of tyrosine kinase inhibitors in chronic phase chronic myeloid leukemia:a patient-based survey on 1133 patients. Leuk. Res., Oxford, v. 39, n. 10, p. 1055-1059, 2015. DOI: $\quad$ https://doi.org/10.1016/j.leukres.2015.07.004. Disponível em:< https://pubmed.ncbi.nlm.nih.gov/26282944/>. Acesso em Acesso em: 12 out. 2020.

CASAGRANDE, V.; SPADElla, M. A.; PINHEIRO, O. L. Desvendando o tratamento da leucemia mieloide crônica. Versão 01. Genebra: Zenodo, 2020. http://doi.org/10.5281/zenodo.3971163

GANESAN, P. et al. Nonadherence to imatinib adversely affects event free survival in chronic phase chronic myeloid leukemia. Am. J. Hematol., New York, v. 86, n. 6, p. 471-474, 2011. DOI: https://doi.org/10.1002/ajh.22019. Disponível em:< https://pubmed.ncbi.nlm.nih.gov/21538468/>. Acesso em 12 out 2020

HAMERSCHLAK, N. et al. Patients perceptions about diagnosis and treatment of chronic myeloid leukemia: a cross-sectional study among Brazilian patients. São Paulo Med. J., São Paulo, v. 133, n. 6, p. 471-479, 2015. DOI: https://doi.org/10.1590/1516-3180.2014.0001306. Disponível em: <http://www.scielo.br/pdf/spmj/v133n6/1516-3180-spmj-1516-3180-2014-0001306.pdf>. Acesso em: 20 out. 2020 .

IVO, R. S. et al. Percepção materna e construção de um material educativo sobre fototerapia. Rev Enferm UFPE Online, v. 11, n. 3, p. 1207-15, 2017/03 2017. DOI: 10.5205/reuol.10544-93905-1-RV.1103201711

Disponível

em:<http://www.revista.ufpe.br/revistaenfermagem/index.php/revista/article/view/9928/pdf_2606

Acesso em: 12 out. 2020.

JABBOUR, E. J. et al. Patient adherence to tyrosine kinase inhibitor therapy in chronic myeloid leukemia. Am. J. Hematol., New York, v. 87, n. 7, p. 687-691, 2012. DOI: https://doi.org/10.1002/ajh.24275. Disponível em:< https://pubmed.ncbi.nlm.nih.gov/22473898/>. Acesso em 18 out. 2020.

MEDEIROS, J. R. R. et al. Validação de tecnologia educativa para cuidado em hemodiálise. Rev. Enferm. UFPE Online, Recife, v. 10, n. 11, p. 3927-3934, 2016. DOI: 10.5205/reuol.9881-87554-1-EDSM1011201614. Disponível em: <http://www.revista.ufpe.br/revistaenfermagem/index.php/revista/article/view/10381/pdf_11314>. Acesso em: 18 out. 2020.

MORISKY, D. E. et al. Predictive validity of a medication adherence measure in an outpatient setting. J. Clin. Hypertens, Greenwich, v. 10, n. 5, p. 348-354, 2008. DOI: https://doi.org/10.1111/j.1751-7176.2008.07572.x. Disponível em:< https://pubmed.ncbi.nlm.nih.gov/18453793/>. Acesso em 18 out. 2020.

Network, National Comprehensive Cancer. Chronic myelogenous leukemia: NCCN evidence blocks: version 2.2021. Fort Washington: National Comprehensive Cancer Network, 2020.

PASQUALI, L. Psicometria: teoria e aplicações. Brasilia: Editora da Universidade de Brasilia, 1997.

RAMOS, L. M. H.; ARAÚJO R. F. R. Uso de cartilha educacional sobre diabetes mellitus no processo de ensino e aprendizagem. Ensino, Saúde e Ambiente. v. 3, n. 10, p. 94-105. 2017. DOI: https://doi.org/110.22409/esa.v10i3.689. Disponível em:< https://periodicos.uff.br/ensinosaudeambiente_backup/article/view/14771/0>. Acesso em 18 out. 2020.

ROESE, F. M.; FONTANA, E. M.; PEREIRA, K. C. B. Análise da adesão à terapia antineoplásica oral de pacientes atendidos na farmácia de quimioterapia de um hospital público de Mato Grosso do Sul. Rev. ENIAC. Pesqui., Guarulhos, v. 7, $\quad$ n. $1, \quad$ p. 125-141, $2018 . \quad$ Disponível em:< https://ojs.eniac.com.br/index.php/EniacPesquisa/article/view/499/pdf>. Acesso em 18 out. 2020. 
SAKO, M. P. et al. Conhecimento sobre precauções na atenção primária à saúde: validação do instrumento. Rev. Bras. Enferm., Brasilia, v. 71, n. 4, p. 1683-1689, 2018. DOI: https://doi.org/10.1590/0034-7167-2017-0886.

Disponível em: <http://www.scielo.br/pdf/reben/v71s4/pt_0034-7167-reben-71-s4-1589.pdf>. Acesso em: 18 out. 2020.

SANTIAGO, R. F. et al. Avaliação de objeto virtual de aprendizagem sobre pré-natal para adolescentes grávidas na atenção básica. Acta Paul Enferm, São Paulo, n.33, p. 1-12, 2020. DOI: https://doi.org/10.37689/actaape/2020ao0063

Disponível em:<https://www.scielo.br/scielo.php?script=sci_arttext\&pid=S0103-21002020000100419>.Acesso em: 18 out. 2020.

SILVA, A. G. et al. Adesão de pacientes ao tratamento com antineoplásicos orais: fatores influentes. Rev. Baiana Enferm., Salvador, v. 31, n. 1, p. 1-12, 2017. DOI: https://doi.org/10.18471/rbe.v31i1.16428. Disponível em:< https://portalseer.ufba.br/index.php/enfermagem/article/view/16428/14063>. Acesso em 18 out. 2020

SIMONEAU, C. A. Treating chronic myeloid leukemia: improving management through understanding of the patient experience. Clin. J. Oncol. Nurs., Pittsburgh, v. 17, n. 1, p. E13-E20, 2013. DOI: https://doi.org/10.1188/13.CJON.E13-E20. Disponível em: < https://pubmed.ncbi.nlm.nih.gov/23372106/>. Acesso em: 18 out. 2020.

SOUSA, C. S.; TURRINI, R. N. T. Validação de constructo de tecnologia educativa para pacientes mediante aplicação da técnica Delphi. Acta Paul. Enferm.,São Paulo, v. 25, p. 990-996, 2012. DOI: https://doi.org/10.1590/S0103-21002012000600026

Disponível em: <http://www.scielo.br/pdf/ape/v25n6/v25n6a26.pdf>. Acesso em: 18 out. 2020.

STEEGMANN, J. L. et al. European LeukemiaNet recommendations for the management and avoidance of adverse events of treatment in chronic myeloid leukaemia. Leukemia, New Jersey, v. 30, n. 8, p. 1648-1671, 2016. DOI: https://doi.org/ 10.1038/leu.2016.104. Disponível em:< https://pubmed.ncbi.nlm.nih.gov/27121688/>. Acesso em: 18 out. 2020.

\section{(cc) EY}

Este trabalho está licenciado com uma Licença Creative Commons - Atribuição 4.0 Internacional. 